\title{
Foot Control of a Surgical Laparoscopic Gripper via 5DoF Haptic Robotic Platform: Design, Dynamics and Haptic Shared Control
}

\author{
Jacob Hernandez Sanchez ${ }^{1,2}$, Walid Amanhoud ${ }^{2}$ \\ Aude Billard ${ }^{2}$ and Mohamed Bouri ${ }^{1}$
}

\begin{abstract}
Foot devices have been ubiquitously used in surgery to control surgical equipment. Most common applications are foot switches for electro-surgery, endoscope positioning and tele-robotic consoles. Switches fall short of providing continuous control as required for precise use of instruments. We developed a haptic foot interface to provide continuous assistance in surgical procedures. This paper concerns the foot control of simultaneous five degrees of freedom (DoF) of a surgical laparoscopic gripper. We assess systematically precision at controlling position and orientation at the target and closing of the forceps. Our controller provides position:position mapping between the foot and the robotic tool, as well as haptic feedback, compensating for gravity of the lower limb of the operator so as to alleviate fatigue. A dynamic model compensation and closed loop force feedback is used to achieve high transparency and backdrivability. The assistance is based on a novel type of haptic fixtures combining spring-damper with selective dynamic compensation in the direction aligned with the task of grasping, so as to simplify control of certain poses, made difficult due to the coupling between human lower limbs' DoF's. We experimentally evaluated the control strategy with six users on a position control surgical task in simulation. Results show the proposed assistance greatly eases the foot grasping task leading to higher completeness, efficiency, and lower mental and physical load.
\end{abstract}

Index Terms-Foot Manipulation, Robotic Surgery, FootRobot Interaction, Surgery Training, Laparoscopy, Gripper

\section{INTRODUCTION}

We target solo robotic surgery scenarios, in which the surgeon does not rely on an assistant and can control more than two instruments simultaneously. This may decrease the number of operating team members needed all at once in a surgical room. These people can then be re-allocated to other tasks, thereby increasing the throughput of surgeries made and shortening waiting lists [1]. Furthermore, communication issues and misunderstandings arising when working in a surgical team could be reduced [2]. Attempts for solo surgery using robotics can vary depending on the intended autonomy of the robotic assistants. For example, a solution could be to strive for fully autonomous robots to collaborate with the main surgeon [1], [3]. Here, we rather follow approaches using direct or semi-autonomous control [4] with emphasis in giving the surgeon the awareness of the interaction (forces and motion of the task) via haptic interfaces. As the surgeon's hands are busy with tasks requiring high dexterity, the

\footnotetext{
${ }^{1}$ REHAssist Group, Swiss Federal School of Technology in Lausanne EPFL, Switzerland. jacob. hernandezsanchez, mohamed.bouridepfl. ch

${ }^{2}$ LASA Laboratory, Swiss Federal School of Technology in Lausanne EPFL, Switzerland. walid.amanhoud, aude.billardeepfl.ch
}

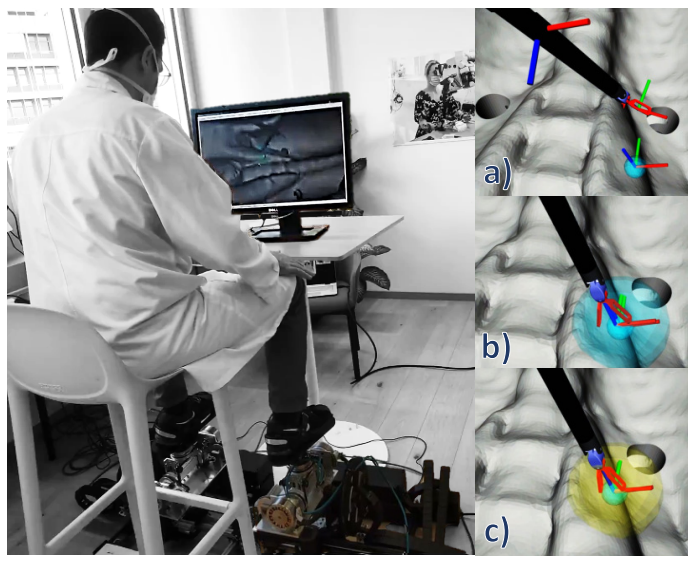

Fig. 1: Foot position control of a surgical gripper assisted by a haptic shared control, performed by a surgeon in the Swiss Foundation for Innovation and Training in Surgery (SFITS), Geneva. When (a) a target appears inside the simulated torso, the foot platform operator (b) moves the too to reach and align with the target both in position (three translations) and orientation (and then a blue visual cue appears), before performing a task of (c) grasping (and then a yellow visual cue appears). When there is a successful alignment and grasping, a new target appears after three seconds.If the user looses the alignment, it should open the gripper again and try again the task. We designed a shared control strategy for the onset of grasping. When the grasping gestures starts, the autonomous control renders a haptic virtual fixture in the foot that selectively eases the grasping, while increasing the resistance for foot gestures related to the positioning of the tool. Furthermore, the surgical robot takes over the position control of the tool while the operator performs the grasp.

additional instruments will then be controlled by the feet. Controlling dexterously an additional instrument with the foot may be very demanding. Robotic assistance is hence desirable to supplement human control. This paper presents the design of a five degrees of freedom foot haptic interface to control a robotic gripper and a shared control strategy to ease its operation. This gripper may act as a laparoscopic retractor clearing the operating view in urological, gynecological or visceral surgery [5].

Foot interfaces have been part of surgical operating rooms for many years, albeit not for performing manipulative tasks. Most medical foot interfaces consist of switches composed of one or a few push buttons, used typically, in on/off mode or to clutch. This can also be used for diathermy to enable an electrically heated probe via two (color-coded) foot switches. These are placed in front of the surgeon, who operates them with a single foot while being in a standing position [6] Tele-robotic surgery consoles can use up to seven switches simultaneously for multiple non concurrent functions (e.g., instrument toggling, camera focus, electrosurgery, clutch, etc) [7]. Similarly, robotic endoscope holders like ViKy, 
AESOP, RoboLens and HIWIN normally require from six to eight foot switches on the floor to control only three degrees of freedom in positioning [8], [9].

Switches fall short of providing required continuous support for solo surgery. To address this need, research efforts have been placed in trying to improve the foot interaction by reading its gestures in multiple degrees of freedom (DoF) and in a continuous manner, aiming at more complex tasks. Tasks considered to date include target reaching and path following tasks [8], [10]-[12], endoscope positioning [9], [13] or holding and supporting tasks [14]. These tasks require at most four and often less degrees of freedom. To both position and grasp using surgical instrument, constrained by a trocar point, it is required to have at least five simultaneous degrees of freedom or DoF (e.g. three rotations + insertion + grasping). The challenge lies then in simultaneously controlling all these degrees of freedom with a single foot. Most foot platforms are limited in DoF (from one to four) [15] [16] [17], and have overlooked the need for controllable force rendering (i.e., no active haptic feedback) [8], [18]-[20], which limits the complexity of the human-robot interaction. Tele-operation using foot position based control via optical markers or inertial measurement units have been associated with fatigue, as it requires users to hold their lower limb in the air [21], [22] (the leg represents around $17.6 \%$ of the body weight in adults [23], [24]). For this reason, isometric or elastic-isometric devices have gained special interest. These platforms require minimum motion and use pressure [25], [26] or force input signals [8], [12] as gestures. As a result, the telemanipulator is commanded in velocity based mode (joystick like mode) with a speed proportional to the input signal. The disadvantage of this mode of control is that the robot position may drift if the input doesn't come back to zero. Also, most of these devices are passive and not easy to change the stiffness of the springs [8], [27]. In this paper we propose an active -position based- haptic interface in five degrees of freedom. Additionally, we propose a solution to the problem of fatigue by including the gravity dynamics of the leg in the control implementation. Thereby, we encourage direct kinematic control between the foot and the robotic arm (position:position) by alleviating the physical load. We seek users to be able to control with our foot interface both reaching, aligning and grasping of a desired target. Two challenges arise: these gestures present bio-mechanical coupling, specifically between the foot ankle rotations of adduction/abduction and eversion/inversion in the talocrular joint [28] that may result in grasping may be more difficult to achieve, if based solely on visual feedback. To address these challenges, we propose a haptic shared controller to ease the simultaneous grasping of the surgical gripper while controlling also for its pose. Capitalizing on the haptic nature of the foot device presented here, we include an event based vibrotactile signal (c.f., [29]) to convey a message (c.f., [30]) to the human when the grasping assistance is enabled. In section $I I$ we describe the mechatronic design and specifications of the foot platform, in section $I I I$ we describe the low level and high level controllers, in section
$I V$ we describe the experimental evaluation on the task of interest (grasping with the foot). Correspondingly, section $V$ presents the results of a user study. Finally section $V I$ presents the conclusion and outlook.

\section{Foot Platform Design}

\section{A. Requirements and Specifications}

The platform should allow to use a position:position mapping (large enough workspace). More over, the platform should be able to render controllable forces. In that respect, the main requirement in the design of the platform is to have enough torques and forces to generate a wrench that is able to meet the gravity torques of the leg, as well as to render perceivable forces for foot haptics (c.f. [31]-[34]). The maximum torques of a person with $120 \mathrm{~kg}$ body mass were considered. Furthermore, the platform should be able to adapt to different sizes of shoes, be easily attached-detached to the foot. Most importantly the size and shape of the platform should be in-obstructive so as to use two foot platforms at the same time. The list of specifications can be found in table I.

\section{B. Kinematics, Workspace and Hardware Implementation}

The kinematic model of the foot platform is illustrated in Fig.2. This is a hybrid parallel-serial kinematic chain, since the first three joints are in series (prismatic, prismatic, revolute) and the last two joints (revolute, revolute) are the result of a (parallel) differential mechanism (see Fig. 3 down). The differential mechanism was used to be able to have double of the nominal torque when isolated rotations are performed. The realization of the foot platform is illustrated in its different components in Fig.3. The platform has five active degrees of freedom, $z$ vertical motion has not been

TABLE I: Specifications of the foot platform

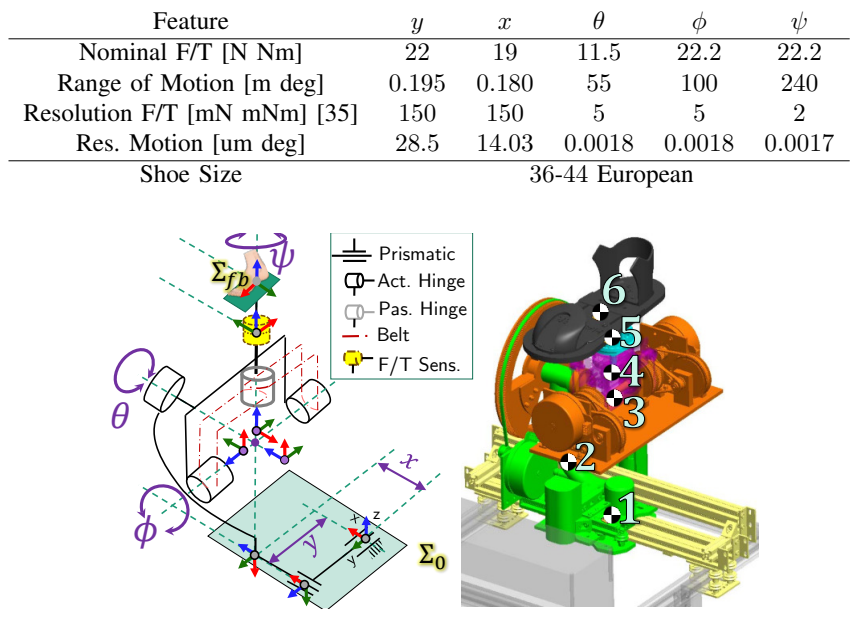

Fig. 2: Left. Kinematic Model of the Foot Platform. The five degrees of freedom are colored in purple. $\Sigma_{0}$ represents the reference frame of the platform, $\Sigma_{f b}$ is the frame of the foot base of the pedal. The last two rotations $(\phi, \psi)$ are possible thanks to a belt driven differential mechanism. This mechanism is illustrated with two active hinges and one passive hinge joint connected via two belts deviated $90^{\circ}$ (n.b.: to understand the working principle refer to Fig.3). Right. Illustration of the 6 kinematic links with different colors. The centers of mass have a white-black icon. 


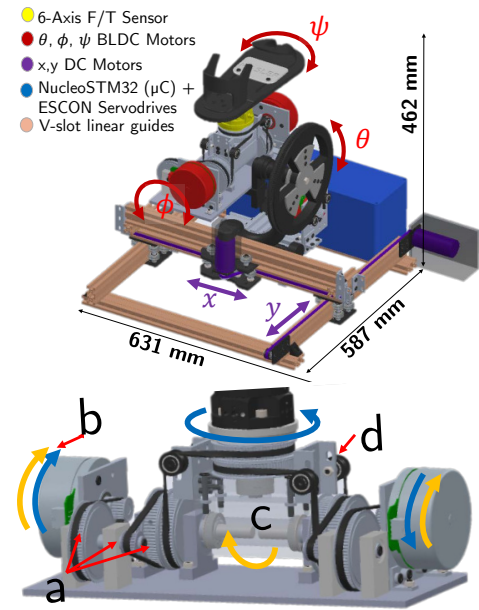

Fig. 3: Mechanical design and mechatronic integration of the foot platform. Up: The prismatic joints (i.e. $x$ and $y$ ) consist in v-slot linear guides, wheels and gantry plates. The motion for $y$ has a fixed $160 \mathrm{~W}$ dc motor and the belt fixed on the moving plate. On the other hand, for the motion in $x$, the $150 \mathrm{~W}$ dc motor is fixed to the moving plate and the belt is fixed to the limits of the frame. The mechanism for $\theta$ consists in a bldc $400 \mathrm{~W}$ motor, and a single stage belt transmission of 12 : 1 . Down: For $\phi, \psi$ the motions are actuated via a differential mechanism of two brushless dc $400 \mathrm{~W}$ motors, with three stage reduction $3.96: 3.96: 1$ (a.) in either side. When the motors rotate in the same direction the $\phi$ motion is enabled, whereas the motors rotate in opposite directions, $\psi$ is actuated (b.). The orthogonal transmission is possible thanks to the use of idler axis in the $\phi$ hinge (c.) and idler pulleys (d.) to deflect the belts $90^{\circ}$. In the base of the pedal, the platform has a 6 axis force-torque sensor [35](e.). Finally, the foot-fender can be adjusted to the different size of shoes

considered as it is the one mostly associated with fatigue [21], [22]). The maximum workspace of the platform, as well as the net foot workspace can be appreciated in Fig. 4.

\section{METHOD}

\section{A. Dynamics and Controls}

The overall control scheme is illustrated in Fig. 5. The control comprises a low level (model based) dynamic compensation and closed loop force feedback for transparency control, and a higher level human-in-the-loop control considering the gravity dynamics of the leg, and a shared control strategy for controlling a surgical tool and rendering of virtual forces.

1) Dynamic modelling: The inverse dynamic model of the foot platform is used to generate feed-forward dynamic compensation torques.

We define the inverse dynamic model of the robotic platform
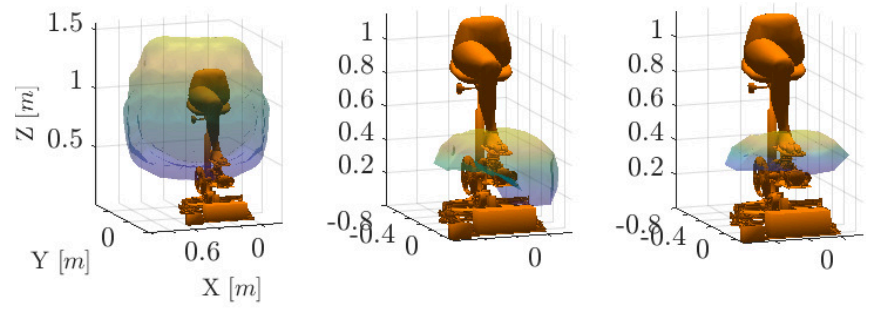

Fig. 4: Left: Human leg's workspace calculated based on the average anthropomorphic data presented in Fig.6. Middle: The workspace of the platform considering joint limits. Right: Net workspace of motion of the foot on the robotic platform. in the joint space like:

$M_{P}\left(q_{P}\right) \ddot{q}_{P}+C_{P}\left(q_{P}, \dot{q}_{P}\right) \dot{q}_{P}+G_{P}\left(q_{P}\right)=\Gamma_{P, i n}-\Gamma_{P, f s}$

where $\boldsymbol{q}_{\boldsymbol{p}}=[y, x, \theta, \phi, \psi]^{T} \in \mathbb{R}^{5}$ represents the vector of coordinates of the platform's state, with $y, x, \theta, \phi, \psi$ being respectively the $\mathrm{y}, \mathrm{x}$ (prismatic joints or translations) and pitch, roll and yaw (revolute joints or rotations). $\boldsymbol{M}_{\boldsymbol{P}}\left(\boldsymbol{q}_{\boldsymbol{P}}\right) \in \mathbb{R}^{5 \times 5}$ is the joint space inertia matrix, which is symmetric and positive definite. $C_{P}\left(\boldsymbol{q}_{\boldsymbol{P}}, \dot{\boldsymbol{q}_{\boldsymbol{P}}}\right) \in \mathbb{R}^{5 \times 5}$ is the centrifugal and coriolis matrix, and $\boldsymbol{G}_{\boldsymbol{P}}\left(\boldsymbol{q}_{\boldsymbol{P}}\right) \in \mathbb{R}^{5}$ is the vector of gravity torques. Finally, $\boldsymbol{\Gamma}_{P, \text { in }} \in \mathbb{R}^{5}$ and $\Gamma_{P, f s} \in \mathbb{R}^{5}$ represent the vector of control and measured torques respectively. In order to implement this model for feed-forward compensation, the joint space inertia matrix and gravity torques were computed like in [36]. The Coriolis and Centrifugal forces factorization proposed by [37] was done to keep skew-symmetry of $\dot{M}_{p}-2 \boldsymbol{C}_{\boldsymbol{p}}$. The inertial information was extracted from the computer aided design (CAD) of platform. The equations were solved in symbolic variables and the explicit expressions of the torques were implemented in the low level control (firmware) in order to achieve a $1 \mathrm{kHz}$ control bandwidth.

2) Closed-loop control with force-feedback: The force torque sensor is used to perform closed loop force control in the joint space [38]. This aims at improving the platform's transparency by inherently compensating the inaccuracies of the inverse dynamic model as well as the nonlinear velocitydependent terms of friction. Furthermore, the force/torque sensor allows to accurately know the interaction forces in the foot pedal.

A proportional control law, $\Gamma_{u, f s}=K_{P, \Gamma} \varepsilon_{P, \Gamma, f s}$, was used for the force feedback. Where $\Gamma_{\boldsymbol{u}, \boldsymbol{f s}} \in \mathbb{R}^{5}$ are the output torques of the force control, $\boldsymbol{K}_{P, \Gamma} \in \mathbb{R}^{5 x 5}$ is a

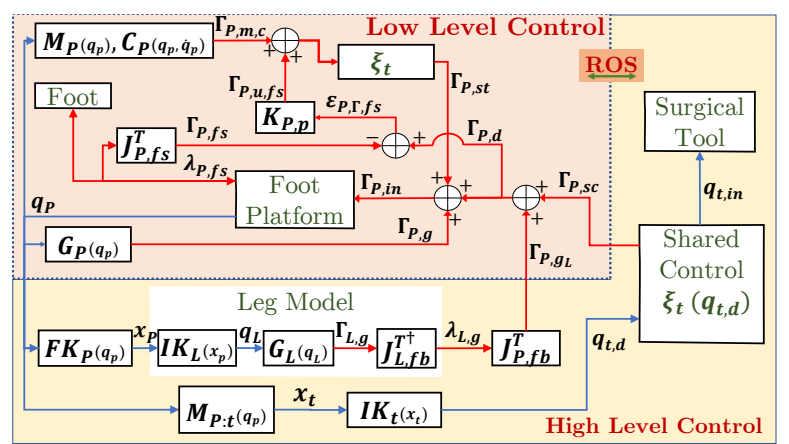

Fig. 5: Overall Control Scheme. A low level firmware control that runs at 1 $\mathrm{kHz}$ takes care of the haptic controllers, inverse dynamic compensation, closed loop force control and joint limits verification. A higher level controller in a PC takes care of the leg inverse kinematics and the shared control that renders torques for the platform and trajectories for the surgical tool. A part of the shared control is done in the firmware. A diagonal matrix $\boldsymbol{\xi}_{t}\left(\boldsymbol{q}_{t, \boldsymbol{d}}\right)$ allows to have a smooth transition between free motion and assisted grasp. Also, this matrix allows selective dynamics compensation and force control in directions related to the task performed by the human (e.g. grasping), while the perceived impedance in higher in the directions where robot is taking over (e.g., positioning) when the shared control is enabled. The communication between the robot and the computer is done via a serial client of Robot Operating System (ROS) at $450 \mathrm{~Hz}$. 


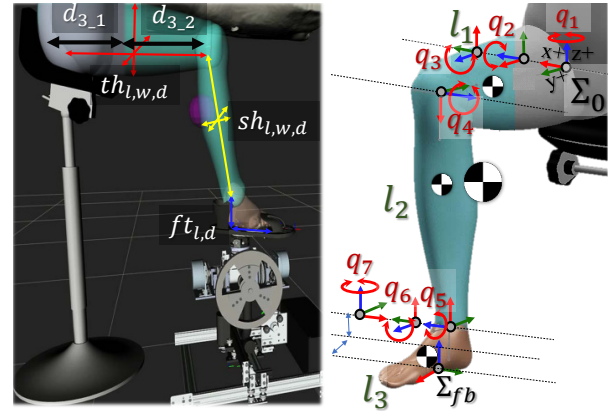

Fig. 6: Model of the leg as a $7 \mathrm{DoF}$ kinematic chain. Left: Visualization of the platform-leg system in RViz. The thigh resting on the chair (color gray, length $d_{3} 1$ ) is assumed constant during the movement. Thereby, only the part of the leg outside of the chair $\left(d_{3_{2} 2}\right)$ is considered in the gravity compensation. The length, width, depth of the thigh $\left(\boldsymbol{t} \boldsymbol{h}_{\boldsymbol{l}, \boldsymbol{w}, \boldsymbol{d}} \in \mathbb{R}^{3}\right)$, shin $\left(s h_{l, w, d} \in \mathbb{R}^{3}\right)$ as well as the ankle to foot tip distance $\left(\boldsymbol{f t} t_{l, d} \in \mathbb{R}^{2}\right)$ of the user are all considered. The ratio of these distances with respect to the baseline of the original CAD, are used to scale the volume of each link w.r.t the center of mass to have a realistic visualization. By changing these parameters, as well as varying the position of the chair as a planar joint in Rviz, the leg model is adapted for every user, and a quick kinematic validation is done at the beginning of the experiment. This procedure has to be done only once. Right: Kinematic chain with joints and links, as well as the positions of the centers of mass of the links and the net center of mass (big white-black icon). The positions of the centers of mass as well as the mass of each link are found based on anthropomorphic data as a function of the body weight [23], [24].

positive-definite matrix with non-negative diagonal entries corresponding to the gains of the control. On the other hand, $\varepsilon_{P, \Gamma, f s} \in \mathbb{R}^{5}$ is the vector of torque errors: $\varepsilon_{P, \Gamma, f s}=$ $\Gamma_{P, d}-\Gamma_{P, f s}$. Where $\Gamma_{P, d} \in \mathbb{R}^{5}$ is the vector of net torques applied on the platform considering all controls. On the other hand, $\boldsymbol{\Gamma}_{P, f s} \in \mathbb{R}^{5}$ are the external torques due to the interaction upon the pedal of the robot. These torques are estimated by projecting the measured wrench of the force-torque sensor and using the the geometric jacobian, as $\Gamma_{P, f s}=\boldsymbol{J}_{P, f s}^{T} \lambda_{P, f s}$. Where $\boldsymbol{J}_{P, f s} \in \mathbb{R}^{6 x 5}$ is the jacobian of the force sensor frame (see Fig. 2) with respect to the base link of the robot.

3) Leg gravity compensation: We consider the gravity dynamics of the leg so that the foot platform generates a wrench to alleviate the leg while moving around, namely to reduce fatigue. We modelled the leg as a $7 \mathrm{DoF}$ serial kinematic chain as illustrated in Fig.6. A URDF (Universal Robot Description File) of the leg as a robot and a YAML(YAML Ain't Markup Language) configuration file makes it easy to change the length segment parameters and update the mass characteristics of the leg robot. The control based on the leg position is done online and sent to the platform via a ROS network. The inverse kinematics of the leg assumes that the foot and the pedal are rigidly attached through frame $\Sigma_{f b}$ (see in Fig.2). Specifically by considering the model of the leg, the position of the chair (that can be changed and visualized in RViz) and the known pose of the foot pedal. The inverse kinematics $\left(\boldsymbol{I} \boldsymbol{K}_{\boldsymbol{L}}\left(\boldsymbol{x}_{\boldsymbol{P}}\right): \mathbb{R}^{6} \rightarrow \mathbb{R}^{5}\right.$ in Fig. 5) is computed using a kinematics and dynamics library (KDL) with weighted damped least squares (WDLS) considering joint limits [39]. The input of the inverse kinematics the pose of the foot platform in the cartesian space $x_{P} \in \mathbb{R}^{6}$.
This corresponds to the position and orientation of the frame in the base of the foot pedal (i.e., $\Sigma_{f b}$ ) and is computed using the direct geometric model (i.e., forward kinematics) of the platform $\boldsymbol{F} \boldsymbol{K}_{\boldsymbol{P}}\left(\boldsymbol{q}_{\boldsymbol{p}}\right): \mathbb{R}^{5} \rightarrow \mathbb{R}^{6}$. Thereby, the gravity torques of the leg $\left(\boldsymbol{G}_{\boldsymbol{L}}\left(\boldsymbol{q}_{L}\right) \in \mathbb{R}^{5}\right)$ are computed for the three links of the leg (thigh, shin and foot, see Fig. 6). Once the gravity torques are known, the corresponding wrench is computed by projecting these torques with the damped pseudo-inverse of the transpose of the jacobian matrix of the frame of the base of the foot, i.e. $\boldsymbol{J}_{L, f b}^{T^{\dagger}} \in \mathbb{R}^{6 x 7}$, like so: $\lambda_{L, g}=J_{L, f b}^{T^{\dagger}} \boldsymbol{\Gamma}_{L, g}$., where $\lambda_{L, g} \in \mathbb{R}^{6}$ corresponds to the gravity wrench. As a consequence, once the wrench required in the foot to match the gravity torques of the leg is known, the corresponding needed torques in the platform are computed like so: $\boldsymbol{\Gamma}_{P, g_{L}}=J_{P, f b}^{T} \lambda_{L, g}$, where $\boldsymbol{\Gamma}_{P, g_{L}} \in \mathbb{R}^{5}$ is the torques of the platform and $\boldsymbol{J}_{P, f b}^{T} \in \mathbb{R}^{5 x 6}$ is the transpose of the jacobian of the platform w.r.t $\Sigma_{f b}$.

4) Shared Control: The torques rendered in the platform during the task of reaching and grasping follow this control law: $\Gamma_{P, s c}=\xi_{t} D_{P, n g} \dot{q}_{P}+$ $\left(1-\xi_{t}\right)\left(K_{P, g} q_{P}+D_{P, g} \dot{q}_{P}+V_{P, d s}\right)$, where $D_{P, n g} \in$ $\mathbb{R}^{5 x 5}$ is a damping matrix to smooth the motion of the user while moving freely from target to target. $\boldsymbol{K}_{\boldsymbol{P}, \boldsymbol{g}} \in \mathbb{R}^{5 x 5}$ and $\boldsymbol{D}_{\boldsymbol{P}, \boldsymbol{n} \boldsymbol{g}} \in \mathbb{R}^{5 x 5}$ are stiffness and damping matrices to push the foot to the position where the grasp was initiated. The latter is a virtual fixture so that the user can freely focus in grasping while the robot controls the position of the tool and haptically fix the position of the foot. Finally, $\boldsymbol{V}_{\boldsymbol{P}, \boldsymbol{g}} \in \mathbb{R}^{5}$ is a vector of zero torque in $q_{P}^{i} \in[y, x, \theta, \psi]$ and the vibrotactile signal (see Fig.7 left) $q_{P}^{j} \in[\psi]$, namely the vibration only acts in the joint related to the grasp $(\phi)$ and is zero for the positioning joints (i.e. $y, x, \theta, \psi)$ ). The iterator $i$ corresponds to the positioning joints of the platform and $j$ corresponds to the grasping joint. The grasping assistance is enabled when a threshold of the platform joint for grasping is surpassed (see Fig. 7 right). Finally $\boldsymbol{\xi}_{t}\left(\boldsymbol{q}_{t, d}\right) \in \mathbb{R}^{5 \times 5}$ is a diagonal positive semi-definite matrix that regulates the transition between the grasping assistance and non-grasping assistance. The entry related to the grasping (i.e., $\xi^{j}$ ) is always 1 , as the human is always in control, whereas the alignment entries (i.e., $\xi^{i}$ ) of this matrix are defined as follows: $\xi^{i}=\left\{\begin{array}{ll}\Upsilon_{t^{*}-t^{*}+\Delta t}(t) & \phi \geq \delta \\ 1-\Upsilon_{t^{*}-t^{*}+\Delta t}(t) & \text { otherwise }\end{array}\right.$, where $\delta$ is the grasping threshold, $\Upsilon_{a-b}(t)$ is a scalar function that smoothly transitions from 0 to 1 , from the beginning time $a=t^{*}$ to the desired duration $b=t^{*}+\Delta t$ like so:

$$
\Upsilon_{a-b}(t)= \begin{cases}0 & t \leq a \\ \left.\frac{1}{2}\left(1+\sin \left(\pi \frac{t-a}{b-a}-\frac{\pi}{2}\right)\right)\right) & a \leq t \leq b \\ 1 & t \geq b\end{cases}
$$

The shared control also involves changes in the dynamic compensation so as to make it more difficult to move in the directions not related to grasping (i.e. those for positioning) while the robot has taken over. For this, the feed-forward 


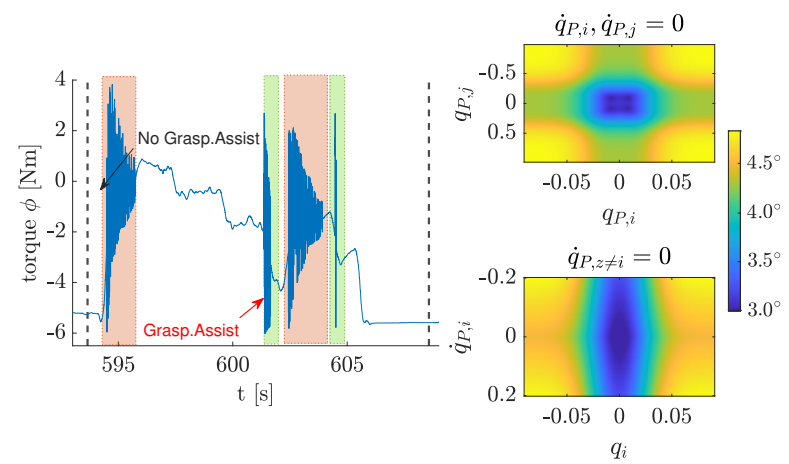

Fig. 7: Grasping assistance. Left: Open loop vibrotactile haptic cues to let the user know when the shared control is enabled or disabled. These are decaying sinusoids, i.e., $V_{P}^{\phi}=a_{v} e^{-d r_{v} t} \sin \left(2 \pi f_{v} t\right)$, where $V^{\phi}{ }_{P}$ is the torque applied in the $\phi$ joint, $a_{v}$ is the amplitude, $d r_{v}$ is the decay rate, $f_{v}$ is the frequency and $t$ is the time. The dashed lines represent the appearance of a new target. A short vibration $\left(a_{v}: 2.5 \mathrm{Nm}\right.$, $\left.d r_{v}: 2, f_{v}: 70 \mathrm{~Hz}\right)$ is rendered when the threshold for grasping intention detection is surpassed and hence the shared control is enabled. Whereas a longer vibration $\left(a_{v}: 2.5 \mathrm{Nm}, d r_{v}: 1.4, f_{v}: 70 \mathrm{~Hz}\right)$ means that the shared control is disabled and the virtual fixtures will disappear. If one vibration is being rendered, the control will wait until the previous one is finished to avoid overlapping. Right: Dynamic threshold for grasping (in $\phi)$. This threshold changes depending on the other degrees of freedom's state to avoid unintentional detection of grasping onset. The equation is $\delta=$ $\sqrt{\sum_{p=0}^{m}\left(\delta_{\max }-b_{t h}\right)\left(a_{t h} e^{\left|\dot{q}_{p}\right|}-\left(\frac{1}{\sigma \sqrt{2 \pi}} e^{\left.-\frac{1}{2}\left(\frac{q_{p}}{\sigma\left(q_{p,+}-q_{p,-}\right)}\right)^{2}\right)}\right)\right.}$ where $m$ is the number of positioning joints (i.e., 4 ), $\delta$ is the angle threshold measured from $0^{\circ}$ in $\phi$, and is limited from $3^{\circ}$ to $\delta_{\max }=5^{\circ}$. $\sigma=0.5$ is the standard deviation of the inverted Gaussian, and $a_{t h}=1.1$ and $b_{t h}=3.0$ are parameters tuned experimentally, and $q_{p,+}$ and $q_{p,-}$ are the limits of the joint $q_{p}$. In the upper image is the example in static condition of one platform's positioning joint against another. The further the positioning joint is from the center of its workspace, the higher is the threshold. In the lower image, the threshold in the phase space of each joint, assuming the others are static. Far from the center of the joint, as the speed increases, the higher the threshold, favouring having grasping assistance when the foot is moving at low speed.

model derived from (1) splits in two:

$$
\left\{\begin{array}{l}
\Gamma_{P, m, c}=M_{P}\left(q_{P}\right) \ddot{q}_{P}+C_{P}\left(q_{P}, \dot{q}_{P}\right) \dot{q}_{P} \\
\Gamma_{P, g}=G_{P}\left(q_{P}\right)
\end{array}\right.
$$

The platform gravity torques $\Gamma_{P, g} \in \mathbb{R}^{5}$ remain the same, while inertia and Coriolis compensation $\Gamma_{P, m, c} \in \mathbb{R}^{5}$ as well as the force control are affected by $\boldsymbol{\xi}_{t}$. As a consequence, the torques of selective transparency control result in: $\Gamma_{P, s t}=\Gamma_{P, g}+\xi_{t}\left(\Gamma_{P, m, c}+\Gamma_{P, c, f s}\right)$, where $\Gamma_{P, s t} \in$ $\mathbb{R}^{5}$ is the state dependent selective dynamic compensation that is complementing the virtual fixtures by adding physical inertia and friction.

\section{EXPERIMENTAL EVALUATION}

The foot platform and control was evaluated through a user study involving 6 participants of $(30 \pm 3)$ years, 2 females and 4 males; naive to the use of the foot platform, from which 2 of them had previous experience with laparoscopic tasks. The task (see Fig.1) was developed in a realistic visualization environment Rviz [41]. A virtual torso (see Fig.8) hosted 30 targets appearing randomly in different parts of its surface. This torso was open source and part of a

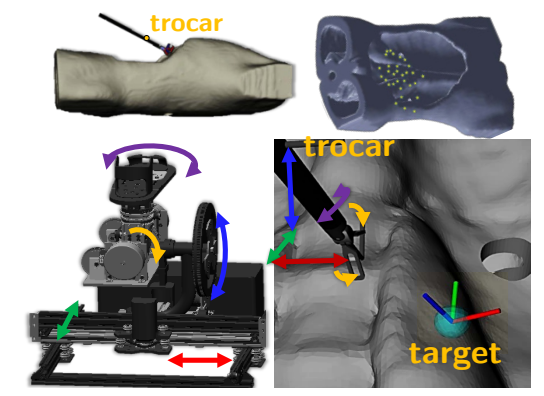

Fig. 8: Up: Set up of the task in the virtual environment, a static realistic torso from [40] hosted the targets in the area indicated with the dots, and trocar constrained robotic surgical tool of 4 dof + grasping was included. The cartesian control of the tool $\left(\boldsymbol{I} \boldsymbol{K}_{t}\left(\boldsymbol{x}_{t}\right)\right)$ is done via KDL inverse kinematics damped least squares. Down: Mapping of the gestures from the foot platform to the motion of the surgical tool. i.e., $x_{t}=M_{P: t}\left(q_{p}\right)$ : $\mathbb{R}^{5} \rightarrow \mathbb{R}^{6}$ (see Fig.5). In this case, $x$ (red line) and $y$ (green line) are mapped in the cartesian $\mathrm{x}$ and $\mathrm{y}$ motions of the tip of the tool respectively; $\theta$ (blue line) is mapped to the $\mathrm{z}$ of the tool, $\psi$ (purple line) is mapped in self rotation of the tool, and finally $\phi$ (yellow line) is mapped in the opening/closing of the gripper. The extra degree of freedom required in the mapping consists in aligning the tool tip $z$ axis pointing towards the trocar point. The workspace of the tool is $(0.30 \mathrm{~m})^{3}$, meaning that the scaling is 1.67 in $x$ and $y$ and 0.3 in $\theta$. Please note how the target's frame is aligned such that the blue axis is pointing towards the trocar point. Thereby, the tool is able to exactly match the target orientation. When the shared control is enabled, the human controls $\phi$, while the autonomy keeps in place $y, x, \theta$ and $\psi$.

realistic phantom [40]. The control of the tool was purely kinematic (no dynamics, no delay). The target was a blue sphere of $14 \mathrm{~mm}$ diameter. The position of this target and the orientation of the other axis were set to randomly change without repeating themselves. The requirements of the task were to have a successful alignment with thresholds of less than $7 \mathrm{~mm}$ position and $7^{\circ}$ orientation, as well as closing the gripper while keeping this alignment (thresholds of $\leq 17^{\circ}$ from the closed position). When these requirements were met, a new target appeared after three seconds. The mapping and the control of the surgical tool are illustrated in Fig.8. The camera of the simulation was set to follow the tip of the tool, and with a fixed zoom adjusted at the beginning of the user study.

Two conditions (with/without shared controlled) were tested twice with each subject. The order of conditions was randomized across subjects. Subjects had 10 minutes to complete each trial, followed by $5 \mathrm{~min}$. rest. At the end of the session, subjects were asked to fill in the questionnaires. At the beginning of the session, the experimenter measured the subject's lower limb lengths to configure appropriately the kinematic model of the simulated leg (see Fig.6).

\section{A. Results and Discussion}

Results are presented in Fig. 9. The Jarque-Bera test was used for normality. For normal distributions, a t-test was used to compare the two conditions (with and without assistance) and Wilcoxon Rank-Sum test was used for the non-normal distributions. Contrasting performance when using shared control and not using it showed statistically significant differences. Shared control improves completeness of the task by $46.6 \%$ (e.g. see Fig.10), median efficiency by $1.7 \%$ and decreases the amount of failed attempts to grasp by $46 \%$. It is 

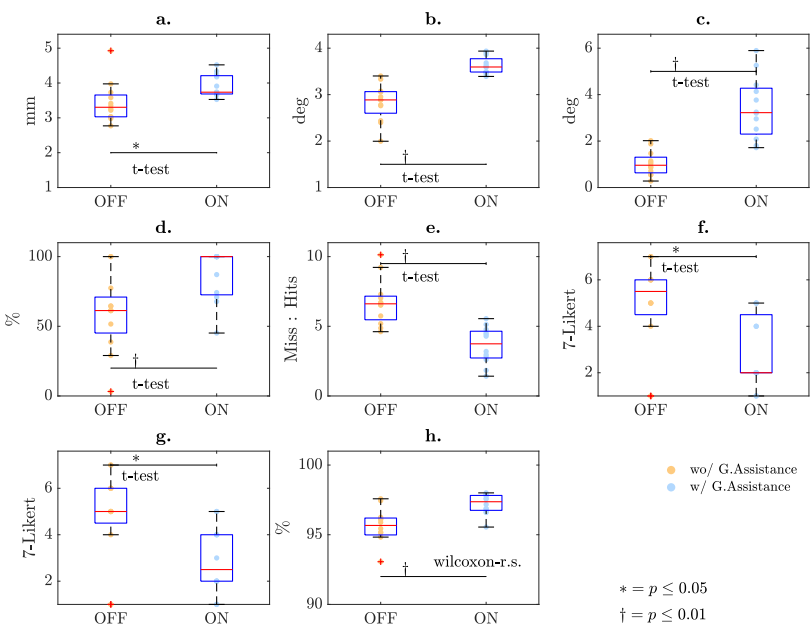

Fig. 9: Metrics of precision ( a. positioning, b. rotational alignment and c. grasping w.r.t to the sphere surface), d. completeness. e. The miss:hits ratio is calculated as the number of times the subject went out of the threshold zone when trying to complete the task, divided by the max. number of targets (i.e., 30). f. and g. correspond to physical and mental load (NASA TLX). h. median efficiency in reaching target to target. Efficiency is defined as scalar function $\nu_{k}=\left(\frac{t_{\Delta k}-t_{\max }}{t_{\max }}\right)$, where $k$ is the target number, $t_{\Delta k}$ is the target to target time.

interesting to see how the precision in positioning, alignment and grasping in this visually controlled task was negatively affected by the shared control $\left(3.91 \pm 0.92 \mathrm{~mm}, 3.62 \pm 0.18^{\circ}\right.$ and $3.42 \pm 1.33^{\circ}$ at the tool level) vs not having shared control $\left(3.44 \pm 0.56 \mathrm{~mm}, 2.83 \pm 0.40^{\circ}\right.$ and $\left.1.04 \pm 0.53^{\circ}\right)$. Most probably because once the grasping assistance was enabled, the users were satisfied with their performance and did not try stepping out of the assistance mode to correct further the error in position/alignment. Nonetheless, the difference is small with respect to the requirements of the task $(<7 \mathrm{~mm}$,
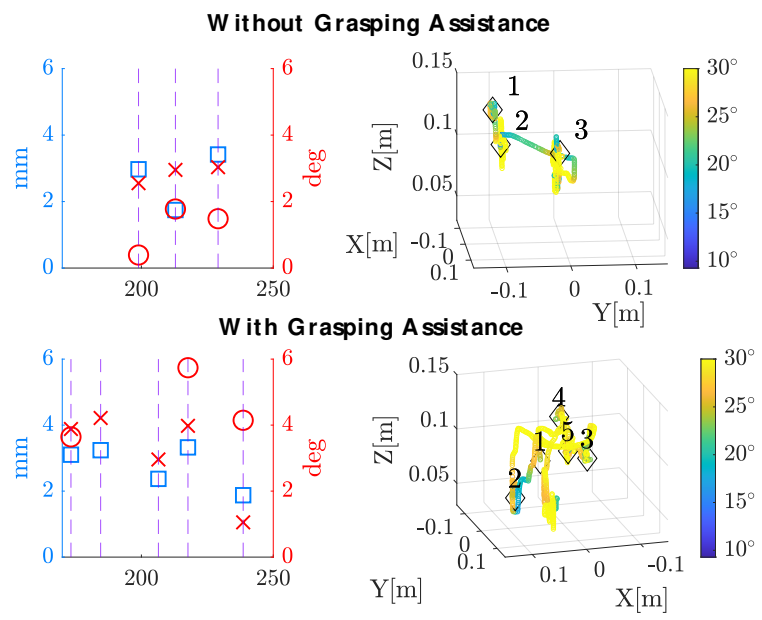

Fig. 10: Example of task performance with and without grasping assistance. Left: Precision in time (the lower the better) of positioning (squares), rotational alignment (crosses) and grasping (circles). The dashed lines represent the transition between targets. Right: trajectories followed by the subject when performing the task illustrated in the pictures of the left. The targets are represented with diamonds. Their number follows the same order as in the left side. The color bar represents the opening and closing of the gripper during the trajectory. $30^{\circ}$ represents a totally open while $0^{\circ}$ represents a totally closed gripper. $<7^{\circ}$ misalignment). An excerpt of the trial in the two conditions is illustrated in Fig.10. Closing the gripper while keeping the alignment without shared control resulted in more number of failed grasps (70 out of 10 successful grasps). The shared control decreased this number to 40 out of 10. This is because it attenuates the coupling between the degrees of freedom. This is evident in the attached video and in Fig.10. For comparison, other foot pos.:pos. systems (c.f. [21]) have reported precision of minimum $13 \pm 46.40$ $\mathrm{mm}$. On the other hand, the shared control helped to reduce physical and mental workload from $4.9 \pm 2.02$ to $2.92 \pm 1.56$, and $4.83 \pm 1.99$ to $2.92 \pm 1.44$ in a 7 -point likert scale, hence reduction of $40 \%$. This means that users perceived the shared control to facilitate the grasping and lower fatigue. For comparison, our system achieves similar mental and physical load as reported for foot-joysticks (i.e., pos.:vel. mapping, c.f. [12]: $2.97 \pm 1.06$ and $3.76 \pm 0.92$ when converted to a 7 point likert scale), while achieving better precision (lower than in [12]: $\sim 5 \mathrm{~mm}$ during a $4 \mathrm{DoF}$ rate-controlled task). In summary, our foot platform has been validated for positioning and grasping tasks requiring pos.:pos. mapping. Indeed, the active haptic feedback, higher number of DoF, transparency control and leg compensation, as well as no dead-zones or position drift, makes our foot platform more versatile than existing interfaces (c.f. [8], [15]-[20]). Finally, during the task, the force tracking $\left(\boldsymbol{\Gamma}_{\boldsymbol{P}, \boldsymbol{f s}}\right.$ vs $\left.\boldsymbol{\Gamma}_{\boldsymbol{P}, \boldsymbol{i n}}\right)$ resulted in maximum root mean squared error of 3.33 and $0.44 \mathrm{mN}$ in $x$ and $y$ (i.e., force), and 190, 75 and $140 \mathrm{mNm}$ in $\theta, \phi$ and $\psi$ (i.e., torque) respectively. This validates the force control.

\section{CONCLUSION AND OUTLOOK}

Results show that subjects, even though naive to control robots with their feet, were capable of manipulating successfully the $5 \mathrm{DoF}$ of the foot platform to reach, align and grasp targets. As expected, the use of the shared control improved significantly performance. Even though users were able to achieve the task with a good level of precision $(3.91 \pm 0.92 \mathrm{~mm})$, most subjects had trouble completing all the targets when not supported by the shared control. This is mainly due to the difficulty of closing the gripper without loosing the alignment. Grasping assistance improved completeness and efficiency; it also reduced physical and mental workloads. These results are promising and present evidence that robotic assistance can reduce fatigue in dexterous position:position foot control. This evaluation was conducted using only kinematic information without considering contact. This said, we believe that rendering the contact information is also important and will be part of our next investigation. The video that accompanies the paper (https://www youtube.com/watch? $\left.v=5 E Y p W x A W y o M \& t=70 \mathrm{~s} \& a b \_c h a n n e l=L A S A\right)$ shows an implementation of our robotic setup with a real gripper.

\section{ACKNOWLEDGMENTS}

The authors thank the Hasler Foundation for their support, and the Swiss Foundation for Innovation and Training in Surgery. 


\section{REFERENCES}

[1] F. Setti, E. Oleari, A. Leporini, D. Trojaniello, A. Sanna, U. Capitanio, F. Montorsi, A. Salonia, and R. Muradore, "A Multirobots Teleoperated Platform for Artificial Intelligence Training Data Collection in Minimally Invasive Surgery," in 2019 Int. Symp. Med. Robot. ISMR 2019. Institute of Electrical and Electronics Engineers Inc., may 2019.

[2] S. A. Elprama, K. Kilpi, P. Duysburgh, A. Jacobs, L. Vermeulen, and J. Van Looy, "Identifying barriers in telesurgery by studying current team practices in robot-assisted surgery," in Proceedings of the 2013 7th International Conference on Pervasive Computing Technologies for Healthcare and Workshops, PervasiveHealth 2013, 2013, pp. 224 231.

[3] G. De Rossi, M. Minelli, A. Sozzi, N. Piccinelli, F. Ferraguti, F. Setti, M. Bonfe, C. Secchi, and R. Muradore, "Cognitive Robotic Architecture for Semi-Autonomous Execution of Manipulation Tasks in a Surgical Environment," in IEEE Int. Conf. Intell. Robot. Syst. Institute of Electrical and Electronics Engineers Inc., nov 2019, pp. 7827-7833.

[4] F. Ferraguti, N. Preda, M. Bonfe, and C. Secchi, "Bilateral teleoperation of a dual arms surgical robot with passive virtual fixtures generation," in 2015 IEEE/RSJ International Conference on Intelligent Robots and Systems (IROS). IEEE, 2015, pp. 4223-4228.

[5] I. Alkatout, "An atraumatic retractor for interdisciplinary use in conventional laparoscopy and robotic surgery," Minim. Invasive Ther. Allied Technol., vol. 27, no. 5, pp. 265-271, sep 2018. [Online]. Available: https://www.tandfonline.com/doi/full/10.1080/ 13645706.2018.1440244

[6] M. A. Van Veelen, C. J. Snijders, E. Van Leeuwen, R. H. Goossens, and G. Kazemier, "Improvement of foot pedals used during surgery based on new ergonomic guidelines," Surgical Endoscopy and Other Interventional Techniques, vol. 17, no. 7, pp. 1086-1091, 72003.

[7] R. P. Goldberg, M. Hanuschik, H. Hazebrouck, P. Millman, D. Kapoor, J. Zabinski, D. Robinson, D. Weir, and S. J. Brogna, "Ergonomic surgeon control console in robotic surgical systems," U.S. Patent US 8,120,301 B2, feb 21, 2012.

[8] Y. Huang, E. Burdet, L. Cao, P. T. Phan, A. M. H. Tiong, P. Zheng, and S. J. Phee, "Performance Evaluation of a Foot Interface to Operate a Robot Arm," IEEE Robot. Autom. Lett., vol. 4, no. 4, pp. 3302-3309, jul 2019.

[9] E. Abdi, M. Bouri, E. Burdet, and H. Bleuler, "Development and Comparison of Foot Interfaces for Controlling a Robotic Arm in Surgery," in 2018 IEEE International Conference on Robotics and Biomimetics, ROBIO 2018. Institute of Electrical and Electronics Engineers Inc., 7 2018, pp. 414-420.

[10] E. Abdi, E. Burdet, M. Bouri, S. Himidan, and H. Bleuler, "In a demanding task, three-handed manipulation is preferred to two-handed manipulation," Scientific Reports, 2016.

[11] E. Abdi, M. Bouri, J. Olivier, and H. Bleuler, "Foot-controlled endoscope positioner for laparoscopy: Development of the master and slave interfaces," in 4th RSI International Conference on Robotics and Mechatronics, ICRoM 2016, 2017.

[12] Y. Huang, E. Burdet, L. Cao, P. T. Phan, A. M. H. Tiong, and S. J. Phee, "A Subject-Specific Four-Degree-of-Freedom Foot Interface to Control a Surgical Robot," IEEE/ASME Trans. Mechatronics, vol. 25, no. 2, pp. 951-963, jan 2020

[13] Y. Huang, W. Lai, L. Cao, J. Liu, X. Li, E. Burdet, and S. J. Phee, "A Three-limb Teleoperated Robotic System with Foot Control for Flexible Endoscopic Surgery,” jul 2020. [Online]. Available: http://arxiv.org/abs/2007.05927

[14] J. Hernandez Sanchez, W. Amanhoud, A. Haget, H. Bleuler, A. Billard, and M. Bouri, "Four-Arm Manipulation via Feet Interfaces," in American Association for Advancement for Artificial Intelligence (AAAI-FSS), Arlington, Virginia, 2019. [Online]. Available: http: //arxiv.org/abs/1909.04993

[15] A. B. Farjadian, S. Suri, A. Bugliari, P. Doucot, N. Lavins, A. Mazzotta, J. P. Valenzuela, P. Murphy, Q. Kong, M. K. Holden, and C. Mavroidis, "vi-RABT: Virtually Interfaced Robotic Ankle and Balance Trainer," in 2014 IEEE International Conference on Robotics and Automation (ICRA). IEEE, 5 2014, pp. 228-233. [Online]. Available: http://ieeexplore.ieee.org/document/6906614/

[16] C. Wang, Y. Fang, S. Guo, and Y. Chen, "Design and Kinematical Performance Analysis of a 3- R US/ R RR Redundantly Actuated Parallel Mechanism for Ankle Rehabilitation," Journal of Mechanisms and Robotics, vol. 5, no. 4, p. 041003, 72013.
[Online]. Available: http://mechanismsrobotics.asmedigitalcollection. asme.org/article.aspx?doi=10.1115/1.4024736

[17] J. A. Saglia, N. G. Tsagarakis, J. S. Dai, and D. G. Caldwell, "Control Strategies for Patient-Assisted Training Using the Ankle Rehabilitation Robot (ARBOT)," IEEE/ASME Transactions on Mechatronics, vol. 18, no. 6, pp. 1799-1808, 12 2013. [Online]. Available: http://ieeexplore. ieee.org/document/6296716/

[18] J. A. Paradiso, S. J. Morris, A. Y. Benbasat, and E. Asmussen, "Interactive therapy with instrumented footwear," in Extended abstracts of the 2004 conference on Human factors and computing systems - CHI '04. New York, New York, USA: ACM Press, 2004, p. 1341. [Online]. Available: http://portal.acm.org/citation.cfm?doid= 985921.986059

[19] A. Rovers and H. van Essen, "HIM," in Extended abstracts of the 2004 conference on Human factors and computing systems - CHI '04. New York, New York, USA: ACM Press, 2004, p. 1313. [Online] Available: http://portal.acm.org/citation.cfm?doid=985921.986052

[20] E. Abdi, M. Bouri, E. Burdet, S. Himidan, and H. Bleuler, "Positioning the endoscope in laparoscopic surgery by foot: Influential factors on surgeons' performance in virtual trainer," in Proceedings of the Annual International Conference of the IEEE Engineering in Medicine and Biology Society, EMBS, 2017.

[21] M. H. Yamen Saraiji, T. Sasaki, K. Kunze, K. Minamizawa, and M. Inami, "MetaArmS: Body remapping using feet-controlled artificial arms," in UIST 2018 - Proc. 31st Annu. ACM Symp. User Interface Softw. Technol. New York, New York, USA: Association for Computing Machinery, Inc, oct 2018, pp. 65-74. [Online] Available: http://dl.acm.org/citation.cfm?doid=3242587.3242665

[22] M. Komori, T. Terakawa, and I. Yasuda, "Experimental Investigation of Operability in Six-DOF Gesture-Based Operation Using a Lower Limb and Comparison with That in an Upper Limb," IEEE Access, vol. 8, pp. $118262-118272,2020$.

[23] R. Contini, R. J. Drillis, and M. Bluestein, "Determination of Body Segment Parameters," Hum. Factors J. Hum. Factors Ergon. Soc., vol. 5, no. 5, pp. 493-504, 1963.

[24] W. S. Erdmann, "Geometry and inertia of the human body," Acta Phys. Pol. A, vol. 135, no. 5, pp. 961-964, 2019.

[25] M. C. Carrozza, A. Persichetti, C. Laschi, F. Vecchi, R. Lazzarini, P. Vacalebri, and P. Dario, "A wearable biomechatronic interface for controlling robots with voluntary foot movements," IEEE/ASME Trans. Mechatronics, vol. 12, no. 1, pp. 1-11, feb 2007

[26] T. Kawai, M. Fukunishi, A. Nishikawa, Y. Nishizawa, and T. Nakamura, "Hands-free interface for surgical procedures based on foot movement patterns," in 2014 36th Annu. Int. Conf. IEEE Eng. Med. Biol. Soc. EMBC 2014. Institute of Electrical and Electronics Engineers Inc., nov 2014, pp. 345-348.

[27] E. Abdi, M. Bouri, J. Olivier, and H. Bleuler, "Foot-controlled endoscope positioner for laparoscopy: Development of the master and slave interfaces," in 4th RSI International Conference on Robotics and Mechatronics, ICRoM 2016. Institute of Electrical and Electronics Engineers Inc., 3 2017, pp. 111-115.

[28] S. Siegler, J. Chen, and C. D. Schneck, "The three-dimensional kinematics and flexibility characteristics of the human ankle and subtalar joints-Part I: Kinematics." Journal of biomechanical engineering, vol. 110, no. 4, pp. 364-73, 11 1988. [Online]. Available: http://www.ncbi.nlm.nih.gov/pubmed/3205022

[29] K. J. Kuchenbecker, J. Fiene, and G. Niemeyer, "Improving Contact Realism Through Event-Based Haptic Feedback," Tech Rep. [Online]. Available: http://www.cis.upenn.edu/ kuchenbe/pub/ pdf/Kuchenbecker06-TVCG-Realism.pdf

[30] A. Rovers and H. van Essen, "FootIO: Design and Evaluation of a Device to Enable Foot Interaction over a Computer Network," in First Joint Eurohaptics Conference and Symposium on Haptic Interfaces for Virtual Environment and Teleoperator Systems. IEEE, pp. 521-522. [Online]. Available: http://ieeexplore.ieee.org/document/1406992/

[31] D. Southall, "The discrimination of clutch-pedal resistances," Ergonomics, vol. 28, no. 9, pp. 1311-1317, 9 1985. [Online]. Available: http://www.tandfonline.com/doi/abs/10.1080/00140138508963248

[32] D. Abbink and F. van der Helm, "Force perception measurements at the foot," in 2004 IEEE International Conference on Systems, Man and Cybernetics (IEEE Cat. No.04CH37583), vol. 3. IEEE, pp. 2525-2529. [Online]. Available: http://ieeexplore.ieee.org/document/ $1400709 /$

[33] A. Ichinose, Y. Gomikawa, and S. Suzuki, "Driving assistance through pedal reaction force control with consideration of JND," 
in 2013 IEEE RO-MAN. IEEE, 8 2013, pp. 484-489. [Online]. Available: http://ieeexplore.ieee.org/document/6628551/

[34] C. Geitner, S. Birrell, C. Krehl, and P. Jennings, "Haptic Foot Pedal: Influence of Shoe Type, Age, and Gender on Subjective Pulse Perception," Human Factors: The Journal of the Human Factors and Ergonomics Society, vol. 60, no. 4, pp. 496-509, 6 2018. [Online]. Available: http://www.ncbi.nlm.nih.gov/pubmed/29768043http: //journals.sagepub.com/doi/10.1177/0018720818771906

[35] "6-axis Force Torque Sensors for robots." [Online]. Available: https://www.botasys.com/

[36] L. N. Truc, N. V. Quyen, and N. P. Quang, "Dynamic model with a new formulation of coriolis/centrifugal matrix for robot manipulators," J. Comput. Sci. Cybern., vol. 36, no. 1, pp. 89-104, feb 2020.

[37] M. Bjerkeng and K. Y. Pettersen, "A new Coriolis matrix factorization," in Proc. - IEEE Int. Conf. Robot. Autom. Institute of Electrical and Electronics Engineers Inc., 2012, pp. 4974-4979.

[38] C. Carignan and K. Cleary, "Closed-loop force control for haptic simulation of virtual environments," Haptics-e, vol. 1, no. 2, 2000.

[39] K. Khokar, P. Beeson, and R. Burridge, "Implementation of KDL inverse kinematics routine on the atlas humanoid robot," in Procedia Comput. Sci., vol. 46. Elsevier B.V., 2015, pp. 1441-1448.

[40] H. G. Kenngott, J. J. Wünscher, M. Wagner, A. Preukschas, A. L. Wekerle, P. Neher, S. Suwelack, S. Speidel, F. Nickel, D. Oladokun, L. Maier-Hein, R. Dillmann, H. P. Meinzer, and B. P. Müller-Stich, "OpenHELP (Heidelberg laparoscopy phantom): development of an open-source surgical evaluation and training tool," Surg. Endosc., vol. 29 , no. 11, pp. 3338-3347, nov 2015. [Online]. Available: https://pubmed.ncbi.nlm.nih.gov/25673345/

[41] D. Hershberger, D. Gossow, J. F. R. Nov, and undefined 2008, "RViz: 3D visualization tool for ROS.(2008)." 